\title{
Zulliger na avaliação de crianças e adolescentes vitimas de abuso sexual
}

Denise Gelain

André Pereira Gonçalves

Anna Elisa de Villemor-Amaral

\section{RESUMO}

O objetivo deste estudo foi verificar se existe diferença entre crianças e adolescentes vítimas de violência sexual intrafamiliar e não vítimas, nas variáveis autopercepção e relacionamento interpessoal do Zulliger, utilizando a aplicação R-otimizada. Os resultados indicaram que nas variáveis de autopercepção apenas $\mathrm{Sx}+\mathrm{Xy}$ foi marginalmente significativo na discriminação dos dois grupos. Considerando as variáveis que compõem o relacionamento interpessoal, COP foi capaz de discriminar os grupos, sendo que o grupo de não vítimas apresentou maiores médias, enquanto que AG, PHR, SumH foram maiores no grupo de vítimas. Além destas variáveis, foram analisados os conteúdos críticos BI e SX; ambos foram capazes de discriminar os grupos, com maiores médias para o grupo de vítimas. Estes resultados indicam que o Zulliger pode auxiliar na avaliação das características de personalidade de vítimas de violência sexual e contribuir para a discriminação entre vítimas e não vítimas.

Palavras-chave: Medidas projetivas de personalidade; Abuso sexual; Avaliação psicológica.

\section{ABSTRACT}

\section{Zulliger in the assessment of sexually abused children and adolescents}

The aim of this study was to verify if there is a difference between children and adolescent victims and non-victims of intrafamilial sexual violence, in Zulliger variables of self-perception and interpersonal relationship, using the R-optimized application. The results indicated that, among self-perception variables, only $S x+X y$ was marginally significant for group discrimination. Considering the variables that compose the interpersonal relationship, COP was able to discriminate the groups (non-victims presented higher average scores), while in GA, $\mathrm{PHR}, \mathrm{SumH}$, the average scores of the victims group was higher. In addition to these variables, critical contents $\mathrm{BI}$ and SX were analyzed; both were able to discriminate the groups, with higher averages for the group of victims. These results indicate that Zulliger can contribute for assessing personality characteristics of victims of sexual violence and also in discriminating between victims and non-victims.

Keywords: Projective personality measures; Sexual abuse; Psychological assessment.

Dentre os tipos de violência que ocorrem contra crianças e adolescentes está a violência sexual. Segundo o Ministério da Saúde (2002), violência sexual consiste no ato ou interações sexuais em que os ofensores utilizam o estímulo sexual em prol da sua satisfação. Tais estímulos compreendem desde atos sem contato físico (assédio, voyeurismo, exibicionismo, apresentação de imagens a criança ou adolescente) até jogos sexuais com contato físico, tais como carícias, sexo oral e penetração genital ou anal.

A estimativa de prevalência de violência sexual contra crianças e adolescentes demonstra um cenário preocupante e que necessita de medidas para inibir a prática deste crime. Uma revisão sistemática realizada por Barth et al. (2013) analisou 55 estudos, de 24 países, publicados entre 2002 e 2009, que tiveram como público alvo crianças e adolescentes até 18 anos,

\section{Sobre os autores}

D. G.

https://orcid.org/0000-00021990-9658

Universidade São Francisco Campinas, SP

denigelain@terra.com.br

\section{A. P. G.}

https://orcid.org/0000-00022470-4040

Universidade São Francisco Campinas, SP

andregoncalvespsi@gmail.com

A. E. V. A

https://orcid.org/0000-0002-

1815-8194

Universidade São Francisco Campinas, SP

anna.villemor@usf.edu.br

\section{Direitos Autorais}

Este é um artigo de acesso aberto e pode ser reproduzido livremente, distribuído, transmitido ou modificado, por qualquer pessoa desde que usado sem fins comerciais. 0 trabalho é disponibilizado sob a licença Creative Commons CC-BY-NC.

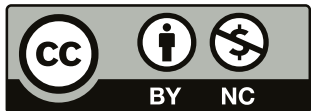




\section{4* INTERACÃO EM PSICOLOGIA}

vítimas de violência sexual. As estimativas de prevalência, nesse estudo variaram de 8 a $31 \%$ para meninas e de 3 a $17 \%$ para meninos. No Brasil de 2011 a 2017 foram registrados 184.524 casos de violência sexual, sendo 58.037 (31,5\%) contra crianças e $83.068(45,0 \%)$ contra adolescentes. A maioria dos registros de abuso em crianças adolescentes ocorreram em meninas, sendo $74,2 \%$ em crianças meninas e $92,4 \%$ em adolescentes meninas (Ministério da Saúde, 2002). Estes dados alarmantes podem ser ainda maiores, pois é estimado que apenas $10 \%$ dos casos são denunciados e consequentemente fazem parte das estatísticas (Faúndes et al., 1998).

Estar exposto a este tipo de violência gera marcas traumáticas e impacta diretamente no desenvolvimento biopsicossocial da vítima (Habigzang e Caminha, 2004; Osofsky, 1995). Como consequências, vítimas de abuso estão mais propensas a desenvolver algum tipo de psicopatologia, sofrer com problemas físicos, emocionais, comportamentais e/ou cognitivos. Neste sentido, tendem a apresentar, com maior frequência, comportamentos de risco, agressivos e delinquentes, dificuldade de concentrar na realização de atividades, queixas somáticas, distúrbios sexuais, depressão, ansiedade e ideação suicida. (Beitchman et al., 1992; Debowska \& Boduszek, 2017; Daigneault et al., 2017; Lewis et al., 2016; Van den Heuvel \& Seedat, 2013).

0 abuso sexual pode ser considerado também um problema social, impactando negativamente a qualidade dos relacionamentos da vítima em vários contextos (e.g. relação com pessoas do mesmo sexo do agressor). Além disso, pode gerar problemas relacionados a aprendizagem e alteração de comportamento no ambiente escolar (Silvaet al., 2013).

0 impacto da violência sexual é ainda maior quando o agressor faz parte do núcleo familiar da vítima, o que caracteriza a violência intrafamiliar (Amazarray \& Koller, 1998; Furniss, 2002; Habigzang \& Caminha, 2004). Ressalta-se que as consequências elencadas variam com relação à intensidade que acometem, desde efeitos menores até transtornos diagnosticáveis (Habigzang et al., 2008a). Em função disso, é sempre pertinente observar a idade do início da violência, a duração, frequência e o grau de agressão; a diferença de idade e proximidade afetiva entre o agressor e as vítimas (Malgarim \& Benetti, 2010).

A gravidade das consequências acarretadas pela violência sexual exige a atuação de profissionais qualificados para proporcionar suporte às vítimas. 0 psicólogo pode assumir um papel importante tanto em acompanhamento terapêutico quanto na realização de avaliações psicológicas. A avaliação psicológica pode fornecer uma compreensão da situação psíquica da pessoa e, por meio das informações obtidas, propor tratamento adequado às vítimas. Pode ser útil, ainda, na tomada de decisão no âmbito jurídico. Porém, apesar de o sistema judicial buscar respaldo técnico dos psicólogos, nem
Denise Gelain, André Pereira Gonçalves e Anna Elisa de Villemor-Amaral

sempre o encontra. Segundo Scortegagna e Villemor-Amaral (2009), alguns vieses ou inadequações são decorrentes, muitas vezes, da escassez de instrumentos com qualidades psicométricas adequadas disponíveis para utilização nesta área.

Habigzang et al. (2008a) enfatizam a premência de haver um escopo maior de instrumentos psicológicos validados que possam ser utilizados no processo de avaliação dos casos de suspeita de violência sexual contra crianças e adolescentes. Tais instrumentos, no processo de avaliação, podem vir a contribuir para o rompimento do ciclo da violência sexual, para o planejamento de intervenções adequadas, assim como para os pareceres psicológicos solicitados pela instância jurídica sobre os casos.

Diversos estudos têm sido realizados com o Rorschach para verificar sua contribuição neste tipo de avaliação (Einbender \& Friedrich, 1989; Friedrich et al., 1999; Saunders, 1991; Ornduff et al., 1999; Scortegagna \& Villemor-Amaral, 2009). Os resultados encontrados nestes estudos indicam que existe diferença entre crianças e adolescentes vítimas de violência sexual e não vítimas principalmente em variáveis como conteúdo sexual ( $\mathrm{Sx}$ ) e sangue (BI), respostas incomuns e conteúdo mórbido (MOR). Os estudos indicam o Rorschach como um instrumento que agrega informações úteis na avaliação de vítimas.

Os resultados obtidos com a utilização do Rorschach levam à hipótese de que o teste de Zulliger, que foi criado com base no Rorschach, poderia também contribuir com a avaliação de crianças e adolescentes vítimas de violência sexual. 0 teste de Zulliger foi criado por Hans Zulliger, mantendo as principais características do Rorschach, como estímulos em manchas de tintas ambíguas, orientação para aplicação, codifıcação e interpretação, porém com um número reduzido de pranchas (Vaz \& Alchieri, 2016; Villemor-Amaral \& Primi, 2009; Zulliger \& Salomon, 1970). Considera-se que o uso do Zulliger no contexto da avaliação de crianças e adolescentes vítimas de abuso seja vantajoso, uma vez que a redução do número de pranchas torna sua aplicação mais rápida e, portanto, menos cansativa para a crianças ou adolescentes, além de agilizar o trabalho do profissional. No Brasil, há dois sistemas disponíveis para aplicação, codificação e interpretação do Zulliger, o Sistema Compreensivo (Villemor-Amaral \& Primi, 2009) e o Sistema Klopfer (Vaz \& Alchieri, 2016). Neste estudo, será adotado o Sistema Compreensivo para codificação das respostas.

Pesquisas mais recentes demonstram que protocolos curtos, com poucas respostas (cinco ou menos) poderiam afetar a qualidade da interpretação dos dados encontrados (Villemor-Amaral et al., 2016). Visto isto, foram iniciados estudos alterando-se as instruções de aplicação e solicitando que a pessoa forneça entre três a cinco respostas por cartão. A mudança nas instruções não mudou, por enquanto, o modo 


\section{H. INTERACÃO EM L PSICOLOGIA}

de análise e interpretação do teste que, nesta pesquisa, se mantiveram os mesmos. A chamada aplicação R-otimizada foi criada por Meyer et al. (2017) para o Rorschach no sistema R-PAS, visando solucionar problemas de normatização do teste decorrentes de uma grande variação no número de respostas por protocolo. Devido aos resultados positivos encontrados nas pesquisas com o Rorschach, se pensou em usar procedimento semelhante no Zulliger, aumentando-se, entretanto, a quantidade média de respostas solicitadas por conta do número reduzido de cartões. A aplicação R-otimizada no Zulliger tem sido estudada e os resultados positivos até o momento encontrados trazem evidências de validade iniciais para este procedimento (Gonçalves et al., 2019; Gonçalves, et al, no prelo; Gonçalves \& Villemor-Amaral, no prelo).

A partir das considerações acima, o objetivo deste estudo foi verificar se existe diferença entre os grupos de crianças e adolescente vítimas de violência sexual intrafamiliar e o grupo de crianças e adolescentes sem histórico deste tipo de violência, considerando as variáveis autopercepção e relacionamento interpessoal do Zulliger, utilizando-se a aplicação $\mathrm{R}$-otimizada. A escolha das variáveis baseou-se em estudos anteriores com o Rorschach que indicaram que a percepção que a pessoa tem de si e os relacionamentos interpessoais, principalmente com pessoas do mesmo sexo que o agressor, são os aspectos mais comumente afetadas neste público. Além disso, foram selecionados os conteúdos de sangue (BI) e Sexo (Sx), também chamados conteúdos críticos, que costumam estar aumentados em situações traumáticas, como variáveis importantes na avaliação de vítimas de abuso sexual (Kendall-Tackett et al., 1993; Leifer et al., 1991; Scortegagna \& Villemor-Amaral, 2009).

\section{MÉTODO}

\section{PARTICIPANTES}

A amostra não probabilística foi selecionada por conveniência e foi composta por 80 crianças e adolescentes divididos em dois grupos distintos. 0 primeiro incluiu 40 vítimas de violência sexual, com idade entre sete e 14 anos $(M=10,68$; $\mathrm{DP}=2,31$ ) sendo 29 do sexo feminino $(72,5 \%)$ e 11 do sexo masculino (27,5\%). Para inclusão dos participantes deste grupo foram considerados os seguintes critérios: a) ser vítima de violência sexual intrafamiliar; b) o perpetrador ser adulto; c) a violência ter sido comprovada conforme documentação que estivesse disponível nas instituições autorizadas para a coleta de dados, tais como: notificação no Conselho Tutelar, notificação no disque 100, Ministério Público, Registro de Ocorrência na Delegacia de Polícia, procedimentos legais de atendimentos médicos nas unidades de saúde ou a médicos
Denise Gelain, André Pereira Gonçalves e Anna Elisa de Villemor-Amaral

legistas, prontuários dessas instituições referentes ao atendimento às vítimas; d) as crianças e adolescentes estarem sendo avaliadas na época da coleta dos dados.

O grupo de crianças e adolescentes não vítimas, foi composto também por crianças de 7 a 14 anos $(M=10,85$; $D P=$ $2,19)$, sendo 29 do sexo feminino $(72,5 \%)$ e 11 do masculino $(27,5 \%)$. Este grupo foi selecionado por professores, levando em conta os seguintes critérios: a) a criança e ou adolescente não ter indícios e história documentada de ter sido vítima de violência sexual; b) não estar em tratamento psicoterápico; c) não apresentar queixas específicas relacionadas a mudanças bruscas de comportamento e processo ensino-aprendizagem.

\section{INSTRUMENTOS}

\section{OUESTIONÁRIO SOCIODEMOGRÁFICO}

Foi constituído por um protocolo com informações sociodemográficas e caracterização da situação da violência sexual, o qual foi aplicado apenas no grupo de vítimas. Contém dados sociodemográficos da criança e/ou adolescente, dados da família, do agressor, dados sobre a violência sexual, revelação, notificação e denúncia.

\section{TESTE DE ZULLIGER NO SISTEMA COMPREENSIVO DE EXNER (VILLEMOR-AMARAL \& PRIMI, 2009]}

É um instrumento composto por três cartões com manchas de tinta impressas, sendo uma acromática, uma policromática e outra em vermelho e preto. A aplicação consiste em duas fases distintas, na primeira os cartões são apresentados. Na segunda fase, chamada de inquérito, os cartões são devolvidos para o examinando que precisa indicar em qual local o objeto foi visto e quais características da mancha lhe deram esta ideia. Os estudos de fidedignidade do teste, por meio do método teste e re-teste e confiabilidade entre avaliadores, indicaram valor acima de 0,70 para concordância entre juízes e correlação acima de 0,60 da primeira com a segunda aplicação, indicando uma associação alta (Villemor-Amaral \& Primi, 2009). O Zulliger neste estudo foi aplicado utilizando o controle do número de respostas seguindo o modelo da aplicação R-otimizada. Nesta aplicação além da orientação "Com que isso se parece?" é solicitado que o examinando forneça entre três e cinco respostas por cartão. Esta aplicação visa evitar protocolos excessivamente curtos que possam afetar negativamente os resultados da avaliação (Villemor-Amaral et al., 2016). Como mencionado acima, os estudos têm demonstrado evidências de validade para o Zulliger com aplicação R-otimizado (Gonçalves et al., 2019; Gonçalves et al., no prelo; Gonçalves \& Villemor-Amaral, no prelo). 


\section{H MTERAC̄OOEM ET PSICOLOGIA}

Denise Gelain, André Pereira Gonçalves e Anna Elisa de Villemor-Amaral

\section{PROCEDIMENTOS}

Foi estabelecido contato com duas instituições que atendem crianças e adolescentes vítimas de violência, sendo um Conselho Tutelar e um serviço integrado de atendimento a psicologia mantido por uma instituição de ensino superior, solicitando autorização para a pesquisa dentro das instituições. Ressalta-se que as crianças e adolescentes frequentavam os serviços para avaliação, mas estavam sob os cuidados familiares. Para a coleta de dados do grupo de não vítimas, foi feito contato com duas escolas estaduais. Todas as instituições estão localizadas em municípios do norte do estado do Rio Grande do Sul. Após as autorizações, a pesquisa foi enviada e aprovada pelo Comitê de Ética em Pesquisa da Universidade de São Francisco sob o CAAE (54111616.2.0000.5514).

Foi realizado um levantamento com a equipe de psicólogos das duas instituições que oferecem serviços de proteção à infância e adolescência por meio dos documentos e prontuários das vítimas de violência sexual, visando selecionar os participantes que preenchiam os critérios de inclusão da pesquisa. Em seguida, os pais ou responsáveis foram contatados para apresentar os objetivos da pesquisa e obter o consentimento para participação no estudo. Para a seleção do grupo de não vítimas foram primeiramente contatadas as escolas estaduais e solicitado aos professores que indicassem os alunos que preenchiam os critérios do estudo. De posse das indicações, foi feito contato com os pais e ou responsáveis para apresentação dos objetivos da pesquisa, e no caso de aceitação, foi assinado o TCLE também em duas vias. A aplicação do teste Zulliger foi realizada individualmente em uma sala disponibilizada pelas instituições.

\section{ANÁLISE DE DADOS}

A análise dos dados foi realizada utilizando o programa estatístico Statistical Package for the Social Sciences (SPSS) 20.0I. Para maior fidedignidade dos dados, $25 \%$ dos protocolos foram sorteados e recodificados por um juiz independente com expertise no teste de Zulliger, sem o conhecimento do grupo de origem. A concordância entre os avaliadores foi avaliada pelo coeficiente Kappa com os valores menores que 0,20 considerados pobres, entre 0,21 a 0,40 suficientes, 0,41 a 0,60 como moderados, 0,61 e 0,80 bons e entre 0,81 a 1,00 excelentes (Landis \& Koch, 1977). A seguir, com intuito de comparar os grupos conforme os códigos do Zulliger selecionados para esta pesquisa, foram realizadas comparações de médias por meio do teste $t$, considerando diferenças significativas as menores que 0,05 e foram calculadas a magnitude das diferenças por meio do $\mathrm{D}$ de Cohen considerando valores acima de 0,30 .

\section{RESULTADOS}

A primeira análise realizada foi a concordância entre os avaliadores por meio do índice Kappa. Para esta análise foi utilizado um juiz especialista, com experiência na utilização e pesquisa com este instrumento. Os resultados estão apresentados na Tabela 1.

\section{Tabela 1. Concordância entre Juízes (KAPPA)}

\begin{tabular}{ccc}
\hline Variáveis & Kappa & Interpretação \\
\hline Localização & 0,96 & Excelente \\
DQ & 0,99 & Excelente \\
Determinantes & 0,93 & Excelente \\
FQ & 0,96 & Excelente \\
Par & 0,93 & Excelente \\
Conteúdo & 0,99 & Excelente \\
Popular & 0,97 & Excelente \\
Zf & 0,85 & Excelente \\
Sum6 & 1,00 & Excelente \\
\hline Nota. Legenda dos códigos do ZSC: Qualidade de \\
desenvolvimento (DQ); qualidade formal (FQ); atividade \\
organizativa (Zf); códigos especiais (Sum6).
\end{tabular}

A concordância entre os avaliadores variou entre 0,85 e 1 e todas classificadas como excelentes. Este resultado permitiu a sequência do trabalho e a realização das análises inferenciais. Na Tabela 2 estão apresentadas as comparações das médias das variáveis que compreendem a autopercepção nos grupos de vítimas de violência sexual e não vítimas.

Pode-se observar que houve diferença marginalmente significativa e de moderada magnitude na variável $A n+X y$ com o grupo de vítimas apresentando as maiores médias. $\mathrm{Na}$ Tabela 3 estão apresentados os resultados da comparação dos grupos nas variáveis relacionadas ao relacionamento interpessoal mais as variáveis $\mathrm{Bl}$ e Sx.

Verifica-se na Tabela 3 que houve diferença significativa entre os grupos de vítimas e não vítimas em sete das 13 variáveis selecionadas. A magnitude destas diferenças variou entre moderada a forte. $\mathrm{O}$ grupo de vítimas apresentou maiores médias em AG, PHR, GHR, SumH, Bl e Sx enquanto o grupo de não vítimas apresentou maior média em COP. 
Tabela 2. Comparação dos escores dos códigos relacionados a variável autopercepção do Zulliger/SC nos dois grupos

\begin{tabular}{|c|c|c|c|c|c|c|c|c|}
\hline Variáveis & Grupos & Min & Máx & Média & DP & $T$ & $p$ & $D$ \\
\hline Egocentrismo & $\begin{array}{c}\text { Vítima } \\
\tilde{N} \text {-vítima }\end{array}$ & $\begin{array}{l}0,00 \\
0,00\end{array}$ & $\begin{array}{l}0,64 \\
0,45\end{array}$ & $\begin{array}{l}0,19 \\
0,15\end{array}$ & $\begin{array}{l}0,15 \\
0,12\end{array}$ & 1,41 & 0,16 & 0,32 \\
\hline Soma de $r$ & $\begin{array}{c}\text { Vítima } \\
\tilde{N} \text {-vítima }\end{array}$ & $\begin{array}{l}0,00 \\
0,00\end{array}$ & $\begin{array}{l}0,00 \\
1,00\end{array}$ & $\begin{array}{l}0,00 \\
0,02\end{array}$ & $\begin{array}{l}0,00 \\
0,16\end{array}$ & $-1,00$ & 0,32 & 0,22 \\
\hline Soma de V & & & & - & - & - & - & \\
\hline FDD & $\begin{array}{c}\text { Vítima } \\
\tilde{\mathrm{N}} \text {-vítima }\end{array}$ & $\begin{array}{l}0,00 \\
0,00\end{array}$ & $\begin{array}{l}2,00 \\
0,00\end{array}$ & $\begin{array}{l}0,07 \\
0,00\end{array}$ & $\begin{array}{l}0,35 \\
0,00\end{array}$ & 1,36 & 0,18 & 0,30 \\
\hline$A n+X y$ & $\begin{array}{c}\text { Vítima } \\
\tilde{N} \text {-vítima }\end{array}$ & $\begin{array}{l}0,00 \\
0,00\end{array}$ & $\begin{array}{l}3,00 \\
2,00\end{array}$ & $\begin{array}{l}0,87 \\
0,52\end{array}$ & $\begin{array}{l}0,88 \\
0,75\end{array}$ & 1,91 & 0,06 & 0,40 \\
\hline MOR & $\begin{array}{c}\text { Vítima } \\
\tilde{N} \text {-vítima }\end{array}$ & $\begin{array}{l}0,00 \\
0,00\end{array}$ & $\begin{array}{l}1,00 \\
1,00\end{array}$ & $\begin{array}{l}0,12 \\
0,12\end{array}$ & $\begin{array}{l}0,33 \\
0,33\end{array}$ & 0 & 1 & 0 \\
\hline
\end{tabular}

Nota. $p \leq 0,01$. Legenda das variáveis do ZSC: índice de Egocentricidade (Ego); respostas reflexos (SumR); respostas de sombreado vista (SumV); respostas de profundidade, distância ou dimensão não relacionadas com o sombreado (FDD); conteúdos de anatomia (An) e raioX (Xy); Proporção entre a quantidade de conteúdo humano $(\mathrm{H})$ e a Soma da quantidade de conteúdo para-humano inteiro [(H)], detalhe humano $(\mathrm{Hd})$ e conteúdo de detalhe para-humano [(Hd)]; respostas com conteúdo mórbido (MOR).

Tabela 3. Comparação dos escores dos códigos relacionados a variável relacionamento interpessoal e variáveis BI e Sx de conteúdo não usual do Zulliger/SC nos dois grupos

\begin{tabular}{|c|c|c|c|c|c|c|c|c|}
\hline Variáveis & Grupos & Min & Máx & Média & $\mathrm{DP}$ & $t$ & $P$ & $D$ \\
\hline COP & $\begin{array}{c}\text { Vítima } \\
\tilde{N} \text { vítima } \\
\end{array}$ & $\begin{array}{l}0,00 \\
0,00 \\
\end{array}$ & $\begin{array}{l}2,00 \\
2,00 \\
\end{array}$ & $\begin{array}{l}0,25 \\
0,50 \\
\end{array}$ & $\begin{array}{l}0,49 \\
0,78 \\
\end{array}$ & $-1,70$ & 0,09 & 0,38 \\
\hline$A G$ & $\begin{array}{c}\text { Vítima } \\
\tilde{N} \text { vítima }\end{array}$ & $\begin{array}{l}0,00 \\
0,00 \\
\end{array}$ & $\begin{array}{l}3,00 \\
1,00 \\
\end{array}$ & $\begin{array}{l}0,55 \\
0,20 \\
\end{array}$ & $\begin{array}{l}0,78 \\
0,40 \\
\end{array}$ & 2,51 & 0,01 & 0,56 \\
\hline PHR & $\begin{array}{c}\text { Vítima } \\
\text { Ñvítima }\end{array}$ & $\begin{array}{l}0,00 \\
0,00\end{array}$ & $\begin{array}{l}3,00 \\
3,00\end{array}$ & $\begin{array}{l}1,07 \\
0,65\end{array}$ & $\begin{array}{l}1,09 \\
0,73 \\
\end{array}$ & 2,03 & 0,04 & 0,46 \\
\hline GHR & $\begin{array}{c}\text { Vítima } \\
\tilde{N} \text { vítima }\end{array}$ & $\begin{array}{l}0,00 \\
0,00 \\
\end{array}$ & $\begin{array}{l}4,00 \\
3,00 \\
\end{array}$ & $\begin{array}{l}1,72 \\
1,32 \\
\end{array}$ & $\begin{array}{l}1,08 \\
0,76 \\
\end{array}$ & 1,90 & 0,07 & 0,43 \\
\hline A & $\begin{array}{c}\text { Vítima } \\
\tilde{N} \text { vítima }\end{array}$ & $\begin{array}{l}0,00 \\
0,00\end{array}$ & $\begin{array}{l}3,00 \\
4,00 \\
\end{array}$ & $\begin{array}{l}1,15 \\
0,87\end{array}$ & $\begin{array}{l}1,09 \\
0,93\end{array}$ & 1,20 & 0,23 & 0,27 \\
\hline$P$ & $\begin{array}{c}\text { Vítima } \\
\tilde{N} \text { Nítima }\end{array}$ & $\begin{array}{l}0,00 \\
0,00 \\
\end{array}$ & $\begin{array}{l}3,00 \\
3,00 \\
\end{array}$ & $\begin{array}{l}0,67 \\
0,57 \\
\end{array}$ & $\begin{array}{l}0,79 \\
0,71 \\
\end{array}$ & 0,59 & 0,56 & 0,13 \\
\hline $\mathrm{Fd}$ & $\begin{array}{c}\text { Vítima } \\
\tilde{N} \text { vítima }\end{array}$ & 0,00 & 1,00 & $\begin{array}{l}0,15 \\
0,12\end{array}$ & $\begin{array}{l}0,36 \\
0,33\end{array}$ & 0,32 & 0,75 & 0,07 \\
\hline SumT & $\begin{array}{c}\text { Vítima } \\
\text { Ñvítima }\end{array}$ & $\begin{array}{l}0,00 \\
0,00\end{array}$ & $\begin{array}{l}1,00 \\
1,00\end{array}$ & $\begin{array}{l}0,07 \\
0,02\end{array}$ & $\begin{array}{l}0,26 \\
0,15\end{array}$ & 1,02 & 0,31 & 0,19 \\
\hline SumH & $\begin{array}{l}\text { Vítima } \\
\text { Ñvítima }\end{array}$ & $\begin{array}{l}0,00 \\
0,00\end{array}$ & $\begin{array}{l}5,00 \\
5,00\end{array}$ & $\begin{array}{l}2,22 \\
1,75\end{array}$ & $\begin{array}{l}1,29 \\
0,95\end{array}$ & 1,87 & 0,06 & 0,42 \\
\hline PureH & $\begin{array}{c}\text { Vítima } \\
\tilde{N} \text { vítima }\end{array}$ & $\begin{array}{l}0,00 \\
0,00\end{array}$ & $\begin{array}{l}5,00 \\
4,00\end{array}$ & $\begin{array}{l}1,22 \\
1,47\end{array}$ & $\begin{array}{l}0,97 \\
0,93\end{array}$ & $-1,17$ & 0,24 & 0,26 \\
\hline PER & $\begin{array}{c}\text { Vítima } \\
\tilde{N} \text { vítima }\end{array}$ & $\begin{array}{l}0,00 \\
0,00\end{array}$ & $\begin{array}{l}3,00 \\
6,00\end{array}$ & $\begin{array}{l}0,60 \\
0,55\end{array}$ & $\begin{array}{l}0,74 \\
1,08\end{array}$ & 0,24 & 0,81 & 0,05 \\
\hline Isolamento & $\begin{array}{c}\text { Vítima } \\
\text { Ñvítima }\end{array}$ & $\begin{array}{l}0,00 \\
0,00\end{array}$ & $\begin{array}{l}0,50 \\
0,70\end{array}$ & $\begin{array}{l}0,21 \\
0,20\end{array}$ & $\begin{array}{l}0,18 \\
0,17 \\
\end{array}$ & 0,24 & 0,81 & 0,06 \\
\hline $\mathrm{Bl}$ & $\begin{array}{l}\text { Vítima } \\
\tilde{N} \text { vítima }\end{array}$ & $\begin{array}{l}0,00 \\
0,00\end{array}$ & $\begin{array}{l}2,00 \\
1,00\end{array}$ & $\begin{array}{l}0,52 \\
0,02\end{array}$ & $\begin{array}{l}0,68 \\
0,15\end{array}$ & 4,54 & 0,00 & 1,01 \\
\hline Sx & $\begin{array}{c}\text { Vítima } \\
\tilde{N} \text { vítima }\end{array}$ & $\begin{array}{l}0,00 \\
0,00\end{array}$ & $\begin{array}{l}3,00 \\
0,00\end{array}$ & $\begin{array}{l}0,32 \\
0,00\end{array}$ & $\begin{array}{l}0,47 \\
0,00\end{array}$ & 4,33 & 0,00 & 0,97 \\
\hline
\end{tabular}

Nota. $\mathrm{p} \leq 0,01$. Legenda das variáveis do ZSC: respostas de movimento cooperativo (COP); respostas de movimento agressivo (AG); respostas com boa representação humana (GHR) e má representação humana (PHR); movimento ativo (a) e passivo (p); respostas com conteúdo de comida (Fd); respostas de sombreado textura (SumT); respostas de conteúdo humano (SumH); respostas com conteúdo humano inteiro (PureH); respostas de conteúdo personalizado (PER); índice de isolamento; conteúdos incomuns de Sangue (BI) e Sexo (Sx). 


\section{H* INTERACÃO EM L PSICOLOGIA}

Denise Gelain, André Pereira Gonçalves e Anna Elisa de Villemor-Amaral

\section{DISCUSSÃO}

0 objetivo deste estudo foi verificar se existe diferença entre os grupos de crianças e adolescente vítimas de violência sexual intrafamiliar e o grupo de crianças e adolescentes que nunca sofreram este tipo de violência nas variáveis autopercepção e relacionamento interpessoal do Zulliger, utilizando-se a aplicação R-otimizada. Foram levantadas hipóteses a partir da literatura da área considerando que sofrer abuso sexual poderia impactar nas variáveis relacionadas a autopercepção, relacionamentos interpessoais e os conteúdos críticos sangue (BI) e Sexo (Sx) (Kendall-Tackett et al., 1993; Leiferet al., 1991; Scortegagna \& Villemor-Amaral, 2009). Os resultados indicaram que houve diferença entre as médias dos grupos nas variáveis de relacionamento interpessoal e conteúdos críticos $\mathrm{Bl}$ e $\mathrm{Sx}$,

Buscar a fidedignidade dos dados por meio da avaliação de juízes independentes é uma prática recorrente nos métodos projetivos. Este procedimento visa limitar o viés do profissional responsável pela codificação das respostas. Os valores de concordância encontrados neste estudo são interpretados como excelentes indicadores de fidedignidade (Landis \& Koch,1977) e estão ao encontro de resultados encontrados em estudos similares (Hosseininasab et al., 2017; Kivisalu et al., 2016; Meyer et al., 2017; Pignolo et al., 2017).

Quando comparada à média de respostas nas variáveis relacionadas a autopercepção, o grupo de crianças e adolescentes vítimas de violência sexual apresentaram maiores médias em $A n+X y$. A variável $A n+X y$ está relacionada com preocupações do indivíduo com o funcionamento corporal; a frequência deste código em excesso pode indicar uma distorção na autoimagem (Exner \& Sendín, 1999). Os resultados deste estudo indicam que as crianças que sofreram violência sexual intrafamiliar demostram percepção da autoimagem mais distorcida quando comparado ao grupo que não sofreu este tipo de violência. Este achado vai ao encontro do estudo realizado por Scortegagna e Villemor-Amaral (2009) utilizando do Rorschach, que encontrou a variável An aumentada em vítimas de violência sexual.

Dentre o grupo de variáveis que avaliam aspectos sobre os relacionamentos interpessoais, as variáveis COP, AG, PHR, SumH foram discriminativas dos grupos. As variáveis de conteúdo crítico Bl e Sx também foram capazes de separar os grupos. 0 grupo de não vítimas apresentou maiores médias em COP, que se refere a capacidade de estabelecer vínculos positivos (Exner \& Sendín, 1999; Meyer et al., 2017). Estes resultados indicam que para a amostra deste estudo, as crianças que não passaram por um episódio de violência apresentam mais capacidade de estabelecerem bons vínculos e manter relacionamentos interpessoais mais saudáveis quando comparados a crianças que foram vítimas de abuso. Nos estudos de Scortegagna e Villemor-Amaral (2009) e de Ornduff et al. (1999), não foram encontradas diferenças significativas em COP.

$O$ código AG está relacionado a percepção dos relacionamentos interpessoais de forma mais destrutiva e agressiva (Exner \& Sendín, 1999; Meyer et al., 2017; Villemor-Amaral \& Primi, 2009). Este código esteve aumentado no grupo de vítimas indicando que este grupo tende a perceber de forma mais agressiva e ameaçadora as relações com outros quando comparado com as crianças e adolescentes não vítimas. Segundo Day et al., (2003) são várias as consequências ao longo da vida para uma pessoa que sofre de violência sexual, dentre elas o medo do agressor e o sentimento de ser ameaçada por pessoas do mesmo sexo que seu agressor o que interfere fortemente na capacidade interpessoal da vítima.

A variável PHR encontra-se significativamente aumentada no grupo de vítimas quando comparado ao grupo de não vítima, o que sinaliza que as vítimas forneceram respostas com percepções mais negativas do ser humano. Dessa forma, verifica-se que essa amostra vítima de violência tende a ter prejuízos potenciais na relação, uma vez que percebe o outro de forma parcial e empobrecida (Exner \& Sendín, 1999; Meyer et al., 2017; Villemor-Aramaral \& Primi, 2009).

As somas de todas as respostas de conteúdos humanos (SumH) esteve aumentada no grupo de vítimas quando comparado ao grupo de não vítimas. Este código precisa ser analisado de forma mais criteriosa do que apenas o resultado bruto. Dentro da soma de conteúdo humano as vítimas de violência sexual apresentaram um maior índice em conteúdos com detalhes humanos e para-humanos e menos com humano Inteiro quando comparado ao grupo de não vítimas, o que indica visão mais parcial, cautelosa, reservada e desconfiada dos outros (Exner \& Sendín, 1999; Meyer et al., 2017; Villemor-Aramaral \& Primi, 2009).

0 grupo de vítimas de violência sexual forneceu mais respostas dos conteúdos críticos $\mathrm{Bl}$ e Sx. Estes resultados corroboram achados anteriores que indicam que vítimas de violência sexual apresentaram no Rorschach aumento dos conteúdos críticos que são mais raros de encontrar nos protocolos de aplicação, dentre eles conteúdos de sangue e sexo (Armstrong \& Loewenstein 1990; Einbender \& Friedrich, 1989; Friedrich et.al., 1999; Scortegagna \& Villemor-Amaral, 2009). Segundo Scortegagna e Villemor-Amaral (2009), as respostas de conteúdo de sangue (Bl) podem ser um reflexo do sentimento da vítima de ser violada, o mesmo podendo-se interpretar de um aumento dos conteúdos sexuais.

Com base nos resultados apresentados, é possível verificar que o Zulliger aplicação R-otimizado pode auxiliar na compreensão das características de personalidade de crianças e 


\section{MLE INTERACÃO EM PSICOLOGIA}

adolescentes que sofreram violência sexual. Neste sentido, algumas variáveis de percepção do relacionamento interpessoal demostraram médias significativamente diferentes nos grupos. Variáveis de conteúdo crítico também foram mais frequentemente observadas nas vítimas, tal como acontece em estudos feitos com o Rorschach. Em síntese, compreende-se que o instrumento foi capaz de discriminar as duas amostras justamente em variáveis relacionais, evidenciando os prejuízos nessa área do funcionamento psicológico das vítimas.

Algumas limitações deste estudo precisam ser consideradas: (a) o tamanho amostral, não permite extrapolar as inferências aqui elaboradas; (b) não equiparação da idade para análises; e (c) falta de informações sobre o tempo, intensidade, gravidade da violência sofrida pela vítima e a proximidade dela com agressor, sendo estas informações relevantes e que precisam ser consideradas em um processo de avaliação neste público. Estudos futuros buscando minimizar as limitações desta pesquisa devem ser realizados. Sugere-se, ainda, que estudos futuros busquem evidências de validade para Zulliger neste público utilizando a relação com instrumentos que mensurem construto similar.

\section{DECLARAÇÃO DAS CONTRIBUIÇÕES DOS AUTORES}

Denise Gelain foi responsável pela concepção do trabalho, coleta de dados, escrita do artigo. André Pereira Gonçalves foi responsável pela análise de dados, escrita do artigo e revisão final. Anna Elisa de Villemor-Amaral foi a responsável concepção do trabalho juntamente com Denise, orientação e supervisão de todo o processo de construção do artigo e também participou da escrita.

\section{DECLARAÇÃO DE CONFLITOS DE INTERESSES}

Os autores declaram que não há conflitos de interesses.

\section{REFERÊNCIAS}

Amazarray, M. R., \& Koller, S. H. (1998). Alguns aspectos observados no desenvolvimento de crianças vítimas de abuso sexual. Psicologia: Reflexão e Crítica, 11(3), 546-555.

Armastrong, J. G., \& Loewenstein, R. J. (1990). Characteristics of patients with multiple personality and dissociative disorders on psychological testing. Journal of Nervous and Mental Disorders, 178, 448-454. https://doi. org/10.1097/00005053-199007000-00006

Barth, J., Bermetz, L., Heim, E., Trelle, S., \& Tonia, T. (2013). The current prevalence of child sexual abuse worldwide: a systematic review and meta-analysis. International Journal of Public Health, 58(3), 469-483. https://doi.org/10.1007/ s00038-012-0426-1
Denise Gelain, André Pereira Gonçalves e Anna Elisa de Villemor-Amaral

Beitchman, J. H., Zucker, K. J., Hood, J. E., DaCosta, G. A., Akman, D., \& Cassavia, E. (1992). A review of the long-term effects of child sexual abuse. Child Abuse \& Neglect, 16(1), 101-118. https://doi.org/10.1016/0145-2134(92)90011-F

Daigneault, I., Vézina-Gagnon, P., Bourgeois, C., Esposito, T., \& Hébert, M. (2017). Physical and mental health of children with substantiated sexual abuse: Gender comparisons from a matched-control cohort study. Child Abuse \& Neglect, 66, 155-165. https://doi.org/10.1016/j.chiabu.2017.02.038

Day, V. P., Telles, L. D. B., Zoratto, P. H., Azambuja, M. D., Machado, D. A., Silveira, M. B., Debiaggi, M., Reis, M. G., Cardoso, R. G., \& Blank, P. (2003). Violência doméstica e suas diferentes manifestações. Revista de psiquiatria do Rio Grande do Sul, 25(1), 9-21. http://dx.doi.org/10.1590/ S0101-81082003000400003.

Debowska, A., \& Boduszek, D. (2017). Child abuse and neglect profiles and their psychosocial consequences in a large sample of incarcerated males. Child Abuse \& Neglect, 65, 266-277. https://doi.org/10.1016/j.chiabu.2017.02.038

Einbender, A. J., \& Friedrich, W. N. (1989). Psychological functioning and behavior of sexually abused girls. Journal of Consulting and Clinical Psychology, 57(1), 155-157. https:// doi.org/10.1037/0022-006X.57.1.155

Exner, J. E., \& Sendín, C. (1999). Manual de interpretação do Rorschach para o sistema compreensivoCasa do Psicólogo.

Faúndes, A., Andalaf, N. J., \& Freitas F. (1998). II Fórum interprofissional sobre o atendimento ao aborto previsto na lei, Femina, 26, 134-138.

Friedrich, W. Einbender, A., \& Carty, P. (1999). Sexually abuse and their Rorschach responses. Psychological Reports, 85, 355-362. https://doi.org/10.2466/pr0.1999.85.2.355

Gonçalves, A. P., Villemor-Amaral, A. E. (no prelo) Zulliger: Aplicação R-otimizado na Avaliação de Características Depressivas. Paidéia.

Gonçalves, A. P., Zuanazzi, A. C., \& Villemor-Amaral, A. E. (2019). Aplicação R-Otimizada no Zulliger: evidências de validade com pacientes depressivos. Avaliação Psicológica, 18(2), 111-120. http://dx.doi.org/10.15689/ ap.2019.1802.16215.01

Gonçalves, A.P., Zuanazzi, A.C., Villemor-Amaral, A.E. (no prelo) Evidências de validade para Zulliger utilizando a aplicação R-otimizada. Arquivos Brasileiros de Psicologia.

Habigzang, L. F., \& Caminha, R. M. (2004). Abuso sexual contra crianças e adolescentes: Conceituação e intervenção clínica. Casa do Psicólogo.

Habigzang, L. F., Corte, F. D., Hatzenberger, R., Stroeher, F., \& Koller, S. H. (2008a). Avaliação psicológica em casos de abuso sexual na infância e adolescência. Psicologia: Reflexão e Crítica, 21(2), 338-344. 


\section{INTERACÃO EM LF. PSICOLOGIA}

Hosseininasab, A., Meyer, G.J., Viglione, D.J., Mihura, J.L., Berant, E., Resende, A.C. \& Mohammadi, M.R. (2017). The effect of CS Administration or an R-optimized alternative on R-PAS variables: A Meta-Analysis of findings from six studies. Journal of Personality Assessment, 1-14. https:// doi.org/10.1080/00223891.2017.1393430

Kendall-Tackett, K. A., Williams, L. M., \& Finkelhor, D. (1993). Impact of sexual abuse on children: A review and synthesis of recent empirical studies. Psychological Bulletin, 113(1), 164-180. https://doi.org/10.1037/0033-2909.113.1.164

Kivisalu, T. M., Lewey, J. H., Shaffer, T. W., \& Canfield, M. L. (2016). An investigation of interrater reliability for the Rorschach Performance Assessment System (R-PAS) in a nonpatient U.S. sample. Journal of Personality Assessment, 98, 382-390. https://doi.org/10.1080/00223891.20 15.1118380

Landis, J. R., \& Koch, G. G. (1977). The measurement of observer agreement for categorical data. Biometrics, 33(1), 159174. https://doi.org/10.3102/0013189X11413260

Leifer, M., Shapiro, J. P., Martone, M. W., \& Kassem, L. (1991). Rorschach assessment of psychological functioning in sexually abused girls. Journal of Personality Assessment, 56(1), 14-28. https://doi.org/10.1207/s15327752jpa5601_2

Lewis, T., McElroy, E., Harlaar, N., \& Runyan, D. (2016). Does the impact of child sexual abuse differ from maltreated but non-sexually abused children? A prospective examination of the impact of child sexual abuse on internalizing and externalizing behavior problems. Child Abuse \& Neglect, 51, 31-40. https://doi.org/10.1016/j.chiabu.2015.11.016

Malgarim, B. G., \& Benetti, S. P. C. (2010). 0 abuso sexual no contexto psicanalítico: das fantasias edípicas do incesto. Aletheia, 33, 123-137.

Meyer, G. J, Viglione, D. J., Mihura, J. L., Erard, R. E., \& Erdberg, R. (2017). Rorschach sistema de avaliação por desempenho manual de aplicação codificação e interpretação e manual técnico (Silva, D. R. \& Miguel, F. K, Trad.; $1^{\text {a }}$ ed). Hogrefe (trabalho original publicado em 2011).

Ministério da Saúde. (2002). Notificação de maus-tratos contra crianças e adolescentes pelos profissionais de saúde: um passo a mais na cidadania em saúde. Brasil, Secretária de Assistência à Saúde.
Denise Gelain, André Pereira Gonçalves e Anna Elisa de Villemor-Amaral

Ornduff, S. R., Centeno, L., \& Kelsey, R. M. (1999). Rorschach assessment of malevolence in sexually abused girls. Journal of Personality Assessment, 73(1), 100-109. https://doi. org/10.1207/S15327752JPA730107

Osofsky, J. D. (1995). The effects of exposure to violence on young children. American Psychologist, 50, 782-788.

Pignolo. C., Giromini. L., Ando. A., Ghirardello. D., Di Girolamo. M., Ales. F., \& Zennaro. A. (2017). An interrater reliability study of Rorschach Performance Assessment System (R-PAS) raw and complexity-adjusted scores. Journal of Personality Assessment 99(6), 619-625.https://doi.org/10 $.1080 / 00223891.2017 .1296844$

Saunders, E. A. (1991). Rorschach indicators of chronic childhood sexual abuse in female borderline inpatients. Bulletin of the Menninger Clinic, 55(1), 48.

Scortegagna, S. A., \& de Villemor-Amaral, A. E. (2009). Autopercepção no Rorschach de vítimas de abuso sexual infantil. Psico, 40(3), 1.

Silva, L. M. P., Ferriani, M. G. C., Beserra, M. A., Roque, E. M. S. T., \& Carlos, D. M. (2013). A escuta de crianças e adolescentes nos processos de crimes sexuais. Ciência \& Saúde Coletiva, 18(8), 2285-2294.

Van den Heuvel, L. L., \& Seedat, S. (2013). Screening and diagnostic considerations in childhood post-traumatic stress disorder. Neuropsychiatry, 3(5), 497.

Vaz, C.E., Alchieri, J.C (2016) Z-teste: coletivo e individual: técnica de Zulliger. Hogrefe.

Villemor-Amaral, A. E., \& Primi, R. (2009). Teste de Zulliger no sistema Compreensivo ZSC: Forma Individual. Casa do Psicólogo.

Villemor-Amaral, A. E., Pianowski, G., \& Carvalho, L, F. (2016). Issues about color, human movement and number of responses in the Zulliger test. Rorschachiana: Journal of the International Society for the Rorschach. 37(2), 95-113. https://doi.org/10.1027/1192-5604/a000068.

Zulliger, H., \& Salomon, F. (1970). El test Z - um test individual y colectivo. Kapelusz.

Data de submissão: 03/09/2019 Primeira decisão editorial: 21/10/2019 Aceite em 22/01/2020 